\title{
New evidence for a role of bone marrow in the pathogenesis of rheumatoid arthritis
}

\author{
Weronika Kurowska, Ewa Kuca-Warnawin
}

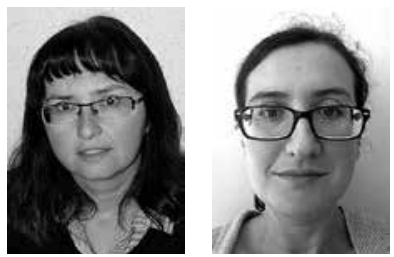

Department of Pathophysiology and Immunology, National Institute of Geriatrics, Rheumatology and Rehabilitation, Warsaw, Poland

Rheumatoid arthritis (RA) is a chronic inflammatory disease leading to joint destruction. Although we know a lot about the pathological processes participating in synovial membrane inflammation (synovitis), synovial hyperplasia and destruction of joint cartilage and bone in established/long-lasting RA, the early pathologic events initiating the disease still remain unclear.

Our and others' data, obtained during the last decade, indicate that bone marrow is an important compartment in RA, where pathological processes from "outside the joint" can occur [1-3]. These studies revealed the presence of cellular infiltrates that replace yellow adipose tissue in bone marrow of RA patients, described on magnetic resonance scans as bone marrow edema (BME). The high frequency (68-75\%) of BME appearance in early RA and its association with painful and aggressive disease course, indicate significance of this phenomena for the pathogenesis of RA. The cellular infiltrates found in RA bone marrow consist of immunological cells that may form aggregates resembling germinal centers in secondary lymphoid organs. Flow cytometry analysis showed an increased number of mononuclear cells and accumulation of activated $\mathrm{T}$ and $\mathrm{B}$ cells in bone marrow of RA patients, in comparison to osteoarthritis patients. The higher levels of early activation markers expressed by $T$ cells, accompanied by elevated concentrations of proinflammatory cytokines (e.g. IL-15) in RA bone marrow, suggest that locally overproduced inflammatory mediators may contribute to T-cell activation and proliferation in situ. $B$ cells can also be activated in the bone marrow microenvironment in RA patients. It was namely shown that B cells isolated from bone marrow of RA patients express functional pathogen recognition receptors (TLR9) and react to microbial components accumulating in this tissue. Furthermore, mesenchymal stromal cells from bone marrow of RA patients possess outstanding properties in supporting aberrant activation of B cells as well as survival of long-life plasma cells. Of note, development of inflammation lesions in bone marrow of RA patients may be facilitated by diminished proportion and/or functional defects of regulatory T cells in this tissue. Importantly, the presence of inflammatory lesions in bone marrow of RA patients, besides having predictive value for disease development, can influence the effectiveness of therapy.

Other investigators revealed that lymphoid aggregates are present in deep marrow areas, distant from the synovial-marrow junction, and BME can be detected soon (within few weeks) at RA onset and also in individuals with undifferentiated arthritis. These findings suggest that inflammation in bone marrow arise independently from pathologic processes operating "inside the joint", and represents an early immunopathological event in RA $[3,4]$.

Intriguingly, data from experimental animal studies showed the enlargement of canals in cortical bone that connect bone marrow to the synovium in the pre-clinical phase of RA. This phenomenon may facilitate migration of precursor cells of synoviocytes and osteoclasts from bone marrow directly to the synovial membrane to excite an inflammatory response and destruction processes. On the basis on these observations the "bone marrow-centered disease model for RA" was proposed [3].

Consistent with this, the appearance of BME was shown to have high value for prediction of bone destruction in RA. Moreover, in a murine model of RA, development of BME was associated with increased differentiation of osteoclast precursors in this tissue [5], and bone marrow of RA patients contains considerable amounts of soluble factors involved in osteoclast differentiation and activation, i.e. tumor necrosis factor $\alpha$ (TNF- $\alpha$ ), RANKL, IL-17 and active, de novo forming osteoclasts $[4,6]$. Interestingly, simultaneous presence of BME and anti-citrullinated protein antibodies (ACPA) raised the 
prediction value of developing RA at 1 year up to 100\% in patients with undifferentiated arthritis, and increased the risk of developing erosive disease in early-stage RA patients [7]. These observations added new information to the previous reported evidence that structural bone damage occurs even in ACPA-positive individuals at risk of developing RA (without detectable inflammation in joints) [8]. The above-described findings prompted the investigators to revise the concept accepted so far that bone damage in RA is an exclusive consequence of synovitis, and suggest a role for bone marrow and ACPA in this process.

Published a few months ago, two new reports provided some evidence that support this notion. It was namely demonstrated that polyclonal ACPA purified from synovial fluid and peripheral blood of RA patients can enhance murine and human osteoclast differentiation in vitro [9]. This stimulatory effect was dependent on the effective citrullination process in osteoclasts, mediated by active peptidyl arginine deiminase enzymes. Transfer of monoclonal ACPA derived from single synovial fluid $B$ cells of patients with RA into mice induced arthralgia and systemic bone loss with no signs of joint inflammation $[9,10]$. The pathogenic effect of human ACPA was associated with interleukin 8 (IL-8) overproduction and was in vivo completely reversed by the IL-8 antagonist reparixin. Most intriguingly, transferred into mice ACPA bound osteoclast precursor cells and osteoclast precursors and osteoclasts residing in bone marrow tissue (not in synovial membrane). These observations led the investigators to hypothesize that osteoclasts and their precursors in bone marrow are the primary target of ACPA, as upon stimulation by ACPA these cells secrete inflammatory mediators and initiate early bone destruction (before development of synovitis). Subsequently, produced in bone marrow inflammatory mediators (i.e. IL-8) may spread via canals in cortical bone into the joint and stimulate the influx of neutrophils and other leukocytes to the synovium. Such a scenario might help answer the long-standing questions regarding why and how ACPA may specifically contribute to joint inflammation, and why the first appearing bone lesions affect cortical bone [8]. It also provides an explanation as to why in ACPA-positive patients with RA, the radiological bone destruction progresses despite achieving persistent remission.

To summarise, the results of recent studies may bring us closer to the understanding of mechanisms triggering pathological events in RA such as bone destruction, which represent nowadays a serious therapeutic challenge. They underline role of bone marrow in the pathogenesis of RA as a site where pathological processes may be initiated, and identify ACPA as an osteoclastogenesis-promoting factor. These findings indicate also new potential targets for therapeutic and preventive interventions interfering with osteoclast activity (IL-8 production/activity, generation of citrullinated proteins which are essential for ACPA stimulatory effect), particularly in ACPA-positive RA patients and ACPA-positive individuals at risk for developing RA.

The authors declare no conflict of interest.

\section{References}

1. Rudnicka W, Burakowski T, Warnawin E, et al. Functional TLR9 modulates bone marrow $B$ cells from rheumatoid arthritis patients. Eur J Immunol 2009; 39: 1211-1220.

2. Kuca-Warnawin E, Burakowski T, Kurowska W, et al. Elevated number of recently activated $T$ cells in bone marrow of patients with rheumatoid arthritis: a role for interleukin 15? Ann Rheum Dis 2011; 70: 227-233.

3. McQueen FM, Ostendorf B. What is MRI bone oedema in rheumatoid arthritis and why does it matter? Arthritis Res Ther 2006; 8: 222.

4. Bugatti S, Caporali R, Manzo A, et al. Involvement of subchondral bone marrow in rheumatoid arthritis: lymphoid neogenesis and in situ relationship to subchondral bone marrow osteoclast recruitment. Arthritis Rheum 2005; 52: 3448-3459.

5. Proulx ST, Kwok E, You Z, et al. Elucidating bone marrow edema and myelopoiesis in murine arthritis using contrast-enhanced magnetic resonance imaging. Arthritis Rheum 2008; 58: 2019-2029.

6. Kurowska W, Kuca-Warnawin E, Maśliński W. Bone marrow - physiological functions and its contribution to the pathogenesis of rheumatoid arthritis. Reumatologia 2010; 48: 247 256.

7. Tamai M, Kawakami A, Vetani M, et al. A prediction rule for disease outcome in patients with undifferentiated arthritis using magnetic resonance imaging of the wrists and finger joints and serologic autoantibodies. Arthritis Rheum 2009; 61: 772-778.

8. Kleyer A, Finzel S, Rech J, et al. Bone loss before the clinical onset of rheumatoid arthritis in subjects with anticitrullinated protein antibodies. Ann Rheum Dis 2014; 73: 854-860.

9. Krishnamurthy A, Joshua V, Haj Hensvold A, et al. Identification of a novel chemokine-dependent molecular mechanism underlying rheumatoid arthritis-associated autoantibody-mediated bone loss. Ann Rheum Dis 2016; 75: 721-729.

10. Wigerblad G, Bas DB, Fernades-Cerqueira C, et al. Autoantibodies to citrullinated proteins induce joint pain independent of inflammation via a chemokine-dependent mechanism. Ann Rheum Dis 2016; 75: 730-738. 